\title{
La expresión oral y escrita en los estudiantes de nivel medio superior mediante el flipped classroom
}

Oral and Written Expression in Upper Secondary Level Students Through the Flipped Classroom

Expressão oral e escrita em alunos do ensino médio durante a sala de aula invertida

\author{
María Elena Zepeda Hurtado \\ Instituto Politécnico Nacional, CECyT No. 11, México \\ mezepedah@ipn.mx \\ https://orcid.org/0000-0001-9764-5013
}

Edgar Oliver Cardoso Espinosa Instituto Politécnico Nacional, Escuela Superior de Comercio y Administración, Unidad Santo Tomás, México eoce@hotmail.com https://orcid.org/0000-0001-7588-9439

Jésica Alhelí Cortes Ruíz Instituto Politécnico Nacional, Centro de Investigaciones Económicas, Administrativas y Sociales, México jacr2709@hotmail.com https://orcid.org/0000-0002-5459-4874 


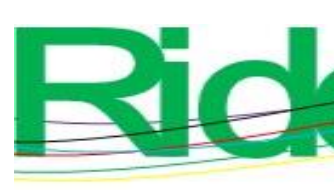

Revista Iberoamericana para la Investigación y el Desarrollo Educativo ISSN $2007-7467$

\section{Resumen}

El objetivo de este estudio fue contribuir con el desarrollo de la expresión oral y escrita mediante el empleo de la estrategia didáctica flipped classroom en estudiantes de nivel medio superior del Instituto Politécnico Nacional. La investigación fue descriptiva con una metodología cuantitativa. Los participantes fueron alumnos que cursaban la unidad Expresión Oral y Escrita II del segundo semestre. El instrumento utilizado fue un cuestionario basado en la escala de Likert. Como parte de los resultados se encontró que el empleo del flipped classroom promueve tres dimensiones: 1) tecnológica: los estudiantes reconocieron una diversidad de recursos tales como el calendario, foros, chats, correo electrónico, autoevaluaciones y consulta de materiales para promover el aprendizaje; 2) comunicativa: los alumnos manifestaron estar de acuerdo con la elaboración de historieta e infografías, analogías, adivinanzas e investigación documental, actividades que favorecieron una participación activa, y 3) prácticas de uso: los alumnos manifestaron que se promovió la comprensión de la estructura del texto literario, así como evaluar y crear una historieta con la estructura literaria.

Palabras clave: aprendizaje, estrategia didáctica, flipped classroom, herramientas tecnológicas.

\section{Abstract}

The objective of this study was to contribute to the development of oral and written expression through the use of the flipped classroom didactic strategy in high school students of the Instituto Politécnico Nacional. The research was descriptive with a quantitative methodology. The participants were students who were studying the Oral and Written Expression II unit of the second semester. The instrument used was a questionnaire based on the Likert scale. As part of the results, it was found that the use of the flipped classroom promotes three dimensions: 1) technological dimension: the students recognized a diversity of resources such as the calendar, forums, chats, e-mail, self-assessments and consultation of materials to promote learning; 2) communicative dimension: the students stated that they agreed with the elaboration of comic strips and infographics, analogies, riddles and documentary research, activities that favored active participation, and 3) usage practices: the students stated that the understanding of the structure of the literary text was promoted, as well as evaluating and creating a short story with the literary structure. 


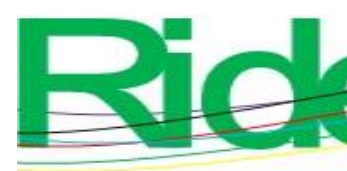

Revista Iberoamericana para la
Investigación y el Desarrollo Educativo
ISSN $2007-7467$

Keywords: learning, didactic strategy, flipped classroom, technological tools.

\section{Resumo}

O objetivo deste estudo foi contribuir para o desenvolvimento da expressão oral e escrita por meio da utilização da estratégia didática de sala de aula invertida em alunos do ensino médio do Instituto Politécnico Nacional. A pesquisa foi descritiva com metodologia quantitativa. Os participantes foram alunos que cursavam a unidade Expressão Oral e Escrita II do segundo semestre. $\mathrm{O}$ instrumento utilizado foi um questionário baseado na escala Likert. Como parte dos resultados, constatou-se que o uso da sala de aula invertida promove três dimensões: 1) tecnológica: os alunos reconheceram uma diversidade de recursos como calendário, fóruns, chats, e-mail, autoavaliação e consulta de materiais para promover a aprendizagem; 2) comunicativa: os alunos afirmaram concordar com a elaboração de histórias em quadrinhos e infográficos, analogias, charadas e pesquisa documental, atividades que favoreciam a participação ativa e 3) práticas de uso: os alunos afirmaram que foi promovida a compreensão da estrutura de o texto literário, bem como avaliar e criar uma história em quadrinhos com a estrutura literária.

Palavras-chave: aprendizagem, estratégia didática, sala de aula invertida, ferramentas tecnológicas.

Fecha Recepción: Marzo 2021

Fecha Aceptación: Septiembre 2021

\section{Introducción}

La globalización ha generado múltiples cambios en diversos ámbitos: en el terreno de la comunicación, la política, en la prestación de servicios y la oferta de productos, entre otros. Dichos cambios han propiciado que las sociedades tengan que modificar sus formas de relacionarse para alcanzar sus objetivos principales de crecimiento y desarrollo a nivel regional y nacional. Lo anterior también trajo consigo una serie de nuevos desafíos para la formación de capital humano, como estar capacitado como para enfrentar las diversas demandas que emergen debido a la gran generación de información y problemáticas a resolver (Núñez y González, 2019). Sin duda es necesario que la educación del siglo XXI se reinvente a fin de desarrollar en los estudiantes diversas capacidades, tales como el pensamiento crítico, la creatividad y la inteligencia emocional (Mendoza, 2019). 


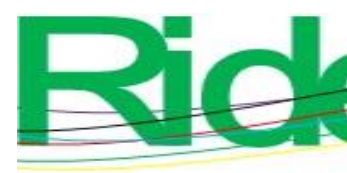

Revista Iberoamericana para la Investigación y el Desarrollo Educativo ISSN 2007 - 7467

De ahí que el tan anhelado cambio de la enseñanza, no centrada en el docente, sino en el estudiante, por supuesto, se trata de un salto pedagógico que implica modificaciones sustanciales en la planeación, implementación y evaluación del proceso educativo. Así, paulatinamente, el profesorado ha intentado abandonar el modelo tradicional de memorización de contenidos y verificación (Santos, Simões y Vieira, 2019). En este sentido, Domínguez y Palomares (2020) indican la preponderancia de fomentar un aprendizaje activo, acompañado del empleo de las tecnologías de la información y comunicación (TIC) que promueva un mayor protagonismo del estudiante y dé lugar a la introducción de metodologías didácticas activas. Se trata, una vez más, de una reforma pedagógica y didáctica con influencia directa en el estudiantado (López, León y Pérez, 2018). Sin embargo, la implementación de metodologías activas se encuentra condicionada por la práctica docente y por los ambientes educativos, por lo que es relevante contextualizarlos a cada nivel (Navaridas y Jiménez, 2016).

De acuerdo con Hinojo, Mingorance, Trujillo, Aznar y Cáceres (2018), el modelo pedagógico flipped classroom está teniendo un gran protagonismo debido a que interrelaciona esta tendencia educativa del aprendizaje activo por parte del alumno con el uso diversificado de las TIC. El flipped classroom también es conocido como aula invertida o inversa suele ser definido como una estrategia didáctica enfocada en dar mayor énfasis a la práctica (Martínez, Nolla, Vidal y de la Torre,2016) ,de este modo, es una metodología didáctica que cambia o invierte la acción de enseñanza expositiva en el salón por parte del docente para enfocarse en llevar a cabo otras actividades centradas en la participación del estudiante: resolución de problemas y análisis de casos, principalmente.

En efecto, el flipped classroom, como su nombre lo indica, se enfoca en invertir los momentos y roles de la enseñanza tradicional (Martínez, Esquivel y Martínez, 2014). Previo a cada sesión el alumno consulta el material multimedia y el tiempo presencial lo ocupa para la revisión del contenido mediante actividades y estrategias activas, es decir, a través de diferentes recursos, el estudiante reconoce los contenidos del tema que se abordará; mientras que la sesión presencial es empleada para la resolución de situaciones prácticas en donde apliquen los conocimientos que permitan desarrollar las competencias en un ambiente de trabajo colaborativo (García y Cremades, 2019).

Aún más, siguiendo a Del Moral (2012) esta metodología didáctica promueve en el estudiante el manejo progresivo de acciones simples a complejas y fomenta el logro tanto de los tres primeros niveles básicos (conocer, recordar, aplicar) como de los de orden superior 


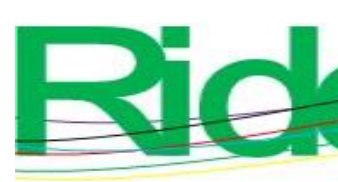

Revista Iberoamericana para la
Investigación y el Desarrollo Educativo
ISSN $2007-7467$

(analizar, evaluar y crear) de la taxonomía de Bloom para conseguir un aprendizaje eficaz, los cuales propician una responsabilidad por el propio aprendizaje.

En el enfoque tradicional, el docente es quien se encarga de la revisión de los contenidos teóricos durante las sesiones; hay poca oportunidad de analizar la información, de realizar ejercicios o prácticas diversas que permitan al alumno abordar los niveles de aprendizaje de mayor profundidad o más complejos, así como comprender, aplicar en diversos contextos o evaluar el objeto de aprendizaje. Esta forma de enseñanza- aprendizaje ha sido sobrepasada por los retos económicos, sociales, culturales y medioambientales que enfrentamos actualmente, de ahí la necesidad de transitar hacia metodologías activas centradas en el alumno, que plantean una perspectiva más ad hoc para atender las problemáticas actuales. Siguiendo esa línea, se seleccionó la metodología flipped classroom para aplicarse en el nivel medio superior y determinar el impacto en la expresión oral y escrita en los alumnos.

Con base en lo anterior, las preguntas que guían la investigación son: ¿en qué consiste la metodología del flipped classroom?; ¿cuáles son las competencias comunicativas desarrolladas a través de esta metodología? y ¿en qué medida el flipped classroom promueve niveles de aprendizaje complejos en los estudiantes?

\section{Fundamentación teórica}

La Organización de las Naciones Unidas para la Educación, la Ciencia y la Cultura [Unesco] (2016) ha posicionado a la educación como pilar fundamental, un objetivo en sí mismo, para el logro de los diversos puntos que conforman la Agenda 2030 para el Desarrollo Sostenible. En dicho documento, en el objetivo 4, se lee: “Garantizar una educación inclusiva y equitativa de calidad y promover oportunidades de aprendizaje permanente para todos" ( $\mathrm{p}$. 7).

En el mismo documento, además de reconocer las necesidades educativas, también se hicieron propuestas políticas para ofrecer una educación de calidad, inclusiva y equitativa:

- Garantizar la adquisición de habilidades, cognitivas, interpersonales y sociales.

- Desarrollar competencias relacionadas con valores y actitudes para llevar una vida saludable y responsable para responder a los desafíos del contexto inmediato y extendido. 


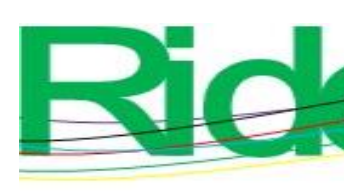

Revista Iberoamericana para la
Investigación y el Desarrollo Educativo
ISSN $2007-7467$

- Ofrecer en todos los niveles educativos aprendizajes de calidad y permanentes.

- Considerar aprendizajes flexibles para su validación y acreditación.

- Aprovechar las TIC.

En el caso particular de México, se consideran las normas y políticas enmarcadas en la Constitución Política de los Estados Unidos Mexicanos (2020), la Ley General de Educación (2019) y La nueva escuela mexicana (2019), en este último, se mencionan las características que conciernen a la educación media superior en lo que respecta al desarrollo de conocimientos, habilidades, aptitudes y actitudes del estudiantado. Para lograrlo, este nivel promoverá valores, a través de una formación humanista, que permitirán el fortalecimiento de competencias profesionales para la generación de capital humano de alto nivel con un desempeño profesional productivo. Por tanto, se pondrá atención principal a :

- El máximo logro de los aprendizajes considerando el contexto y las características propias del estudiante enfatizando el aprendizaje colaborativo y el trabajo en equipo.

- El proceso enseñanza aprendizaje basado en el uso de recursos técnicospedagógicos.

- El aprendizaje, empleo y uso de la tecnología mediante plataformas, herramientas o programas que posibiliten la implementación de las diversas modalidades educativas (presencial, semipresencial o virtual).

- La resolución de problemas a partir de la innovación.

La flipped classroom es una estrategia que considera en su implementación los anteriores puntos, va más allá de proporcionar materiales digitales para que los alumnos los revisen en casa; se enfoca en combinar el empleo de herramientas tecnológicas con técnicas didácticas constructivistas que potencien el desarrollo de competencias. Consecuentemente, para poner en práctica metodologías activas, hay que conceptualizar de manera diferente el aprendizaje y los desempeños del docente y del alumno.

Calvo (2020) establece que el aprendizaje invertido es "un proceso interactivo basado en la comunicación profesor-estudiante, estudiante-estudiante, estudiante-material didáctico y estudiante-medio que potencia la implicación responsable de este último y conlleva la satisfacción y enriquecimiento de docentes y estudiantes”. En tanto que Silva y Maturana (2017) indican que las metodologías activas se centran en el desarrollo de las actividades o experiencias de aprendizaje más que en los contenidos, lo que implica cambios en el profesor, 


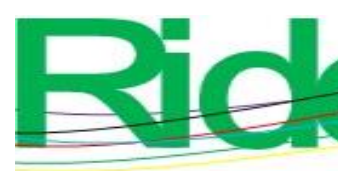

Revista Iberoamericana para la
Investigación y el Desarrollo Educativo
ISSN $2007-7467$

presencial, optimiza la interacción entre docente y estudiante y desarrolla la autonomía en cada alumno.

Específicamente, Bondad, Rice y Pearce (2012) señalan que en el método flipped classroom el uso de vídeos en línea se convierte en un recurso idóneo. Además, recomiendan que dichos videos tengan las siguientes características: breves para su consulta, el contenido debe tener una cantidad manejable de información para que sea interesante y su diseño debe estar ajustado a los estudiantes. De la misma manera, se debe prever cierta interactividad porque mejora la experiencia de aprendizaje (Huang, Chen y Weng, 2012; Verleur, Heuvelman y Verhagen, 2011).

Por consiguiente, tomando en cuenta que el flipped classroom integra ambientes de aprendizaje mediados por las TIC, es relevante identificar, analizar, discernir y seleccionar la información que posibilite el logro de las competencias (Álvarez y Boillos, 2015). Por último, algo que es imposible pasar por alto es que este método fomenta el trabajo colaborativo, el cual, a la larga, permite la formación de actitudes tales como la creatividad, la negociación y toma de decisiones que les sirven a los estudiantes para formar experiencia previa al ejercicio laboral (Álvarez, Herrejón, Morelos y Rubio, 2010).

\section{Literatura previa}

En el trabajo de Melo y Sánchez (2017), los estudiantes participantes reconocieron que la metodología del flipped classroom dio la oportunidad de un aprendizaje más activo y experiencial; la organización y estructura de cada una de las sesiones les permitió hallar la relevancia de los contenidos; el tiempo invertido fue productivo, los videos utilizados y su influencia en el desarrollo de las actividades durante las clases fueron prácticas. Así, $81 \%$ indicó un nivel de satisfacción alto por el empleo de esta metodología.

Simon, Ojando, Avila, Miralpeix, Lopez y Prats (2018), por su parte, hallaron que $96.9 \%$ de los alumnos consideraron que el modelo de flipped classroom es positivo para la formación de sus aprendizajes; $44 \%$ indicó que los materiales fueron de fácil acceso; 60 \% indicó que los materiales didácticos combinan diversos formatos (texto, audio y vídeo); $35.7 \%$ reconoció la utilidad y relevancia de que los materiales y recursos empleados sean en formato digital, y $33.7 \%$ valoró que los materiales respetan su estilo de aprendizaje. Además, $61.2 \%$ reconoció que el aprendizaje es más experiencial y $38.8 \%$ manifestó que se adquieren las habilidades de creación, análisis y evaluación. Por otro lado, $54.1 \%$ identificó que el 


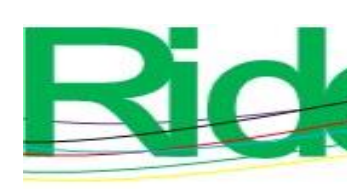

Revista Iberoamericana para la
Investigación y el Desarrollo Educativo
ISSN $2007-7467$

profesor se convierte en una guía para el aprendizaje; $64.3 \%$ consideró que la planeación de las actividades se caracteriza por ser interactiva, es decir, alumnos y maestro participan en la planeación de actividades. Al final concluyeron que el docente emplea una evaluación continua, utiliza la autoevaluación y coevaluación.

Mientras que Schmeisser y Medina (2018) determinaron que en asignaturas de matemáticas y español la metodología del flipped classroom mejoró el rendimiento académico en términos estadísticos. Los recursos tecnológicos empleados fueron el uso de teléfonos inteligentes, tabletas y laptops; mientras que los materiales didácticos usados fueron videos, lecturas y ejercicios. La principal conclusión fue que el aula invertida desarrolló el pensamiento crítico, autonomía y responsabilidad por el aprendizaje.

Cabe destacar que el pensamiento crítico se desarrolla cuando se consultan diversas fuentes en las cuales se analiza y se evalúa la información. Por ejemplo, se valora la confiabilidad de la fuente, se cuestionan las afirmaciones y se contrastan con hechos reales para interpretar y explicar con argumentos.

Por último, los participantes del estudio de García y Cremades (2019) caracterizaron a la metodología del flipped classroom como innovadora y útil, que permite un aprendizaje cooperativo, la socialización y la autonomía. Asimismo, manifestaron que los materiales y las sesiones presenciales les permitieron adquirir contenidos conceptuales, procedimentales y actitudinales. Por tanto, el proceso de aprendizaje involucró los niveles de aprendizaje de la taxonomía de Bloom (citado en Del Moral, 2012).

\section{Objetivo del estudio}

El objetivo de la presente investigación es emplear la estrategia didáctica flipped classroom para desarrollar expresión oral y escrita en los estudiantes del nivel medio superior del Instituto Politécnico Nacional (IPN).

\section{Método}

\section{Tipo de estudio}

Esta investigación tuvo un enfoque cuantitativo con un alcance descriptivo porque se propuso valorar los niveles de desarrollo tanto de la expresión oral como escrita en los alumnos participantes (Hernández y Mendoza, 2018; Namakforoosh, 2011). 


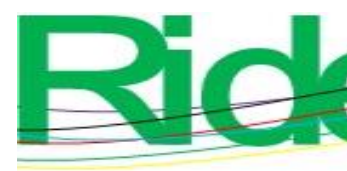

\section{Revista Iberoamericana para la Investigación y el Desarrollo Educativo ISSN 2007 - 7467}

Para lograrlo, se utilizó la estrategia didáctica de flipped classroom en los estudiantes que cursan la unidad de aprendizaje de Expresión Oral y Escrita II (EOYE II), ubicada en el segundo nivel del mapa curricular de la educación media superior. El reto fue que los alumnos abordaran los contenidos teóricos fuera del aula con la finalidad de dar oportunidad a que en la clase presencial se enfocaran en la realización de actividades de tipo práctico que fomentaran el desarrollo de la comunicación escrita y oral en los estudiantes.

Huelga decir que los contenidos de esta unidad de aprendizaje se orientan a abordar temas relacionados con la literatura. Por lo extenso del contenido y lo ambicioso de las competencias a desarrollar, metodologías como el flipped classroom son ideales para detonar el proceso creativo y la elaboración de producciones literarias como la redacción de breves textos expresivos correspondientes a los tres géneros literarios por antonomasia (narrativo, lírico o poesía y dramático o teatro). Así, esta unidad de aprendizaje tiene el objetivo de desarrollar competencias comunicativas que se emplean a lo largo del curso: hablar, escuchar, leer y escribir, las cuales son consideradas competencias instrumentales o genéricas útiles en diversos ámbitos como el personal, académico y laboral o profesional.

Específicamente, para esta investigación se seleccionó la primera unidad programática del curso, denominada "Análisis literario", cuya competencia específica, de acuerdo con el programa de estudios (IPN, 2008), es la siguiente: "Emite juicios críticos sobre los mensajes expresivos a partir del contexto social en el cual surgen" (p. 17); y cuyo resultado de aprendizaje esperado es: "Analiza textos expresivos y determina la estructura del texto literario a partir de las formas de expresión y las figuras literarias” (p. 7).

\section{Participantes}

Los participantes fueron 54, quienes tenían entre 15 y 17 años. En cuanto al género, $32(59 \%)$ correspondieron al género femenino y $22(41 \%)$ al masculino. Se empleó una muestra no probabilística incidental, por lo que los alumnos seleccionados fueron aquellos que estaban inscritos en el segundo semestre.

\section{Intervención didáctica}

El IPN (2008), con miras a fortalecer el trabajo autónomo de los estudiantes, destina 18 horas por semestre para la realización de actividades fuera del aula, en otros ambientes, para fomentar la investigación, trabajo colaborativo o desarrollo de las diversas fases de los 


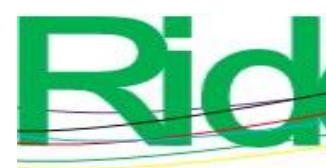

Revista Iberoamericana para la
Investigación y el Desarrollo Educativo
ISSN $2007-7467$

proyectos y así transitar de la teoría a la práctica a través de actividades prácticas. Además, la metodología se fundamenta en estándares de aprendizajes basados en competencias, las cuales se valorarán mediante los resultados del aprendizaje esperado. La evaluación se enfoca a la generación de evidencias que demuestren el saber hacer a través de un desempeño o producto relacionado con el conocimiento aplicado en situaciones que permitan la transferencia a contextos similares o diferentes.

Para aplicar el flipped classroom se consideró la Unidad I del programa, titulada "Análisis literario". Debido a las características de esta unidad, orientadas a la realización de actividades prácticas, se optó por la modalidad tipo taller, los contenidos de tipo teóricos se desarrollaron fuera del aula a través de la revisión de materiales proporcionados y las sesiones presenciales se destinaron al trabajo para consolidar los conocimientos.

En este sentido, la planeación que se implementó fue relevante porque permitió tanto la selección del material digital o elaboración de recursos relacionados con los contenidos de la unidad de aprendizaje, principalmente videos breves, entre 5 y 10 minutos de duración, interesantes, atractivos y disponibles para los estudiantes.

Así, dicha planeación se fundamentó en la propuesta de García y Quijada (2015), cuyos elementos son aplicables para este estudio haciendo algunas adaptaciones y tomando en cuenta los recursos disponibles se consideraron: $a$ ) dimensión tecnológica, $b$ ) dimensión psicopedagógica y $c$ ) prácticas de uso. En la tabla 1 se puede observar la relación entre contenidos, dimensiones, prácticas de uso y la competencia que se consideraron en la planeación realizada. 


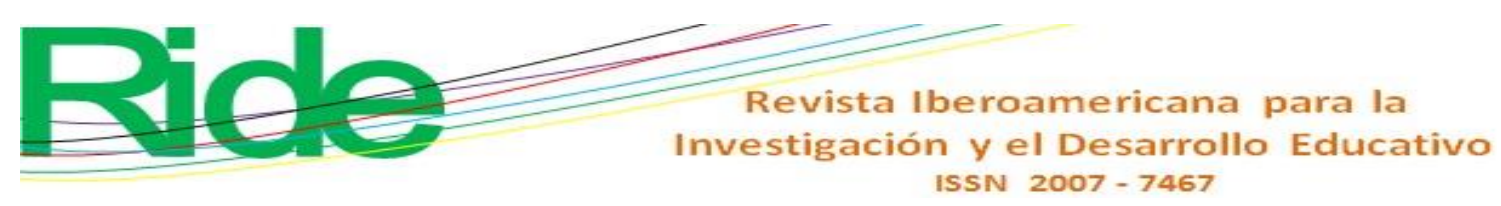

Tabla 1. Planeación unidad I "Análisis literario"

\begin{tabular}{|c|c|c|c|c|}
\hline Contenido & $\begin{array}{l}\text { Dimensión } \\
\text { tecnológica, } \\
\text { recursos } \\
\text { informáticos }\end{array}$ & $\begin{array}{c}\text { Dimensión } \\
\text { psicopedagógica }\end{array}$ & $\begin{array}{c}\text { Prácticas de uso. } \\
\text { Niveles } \\
\text { cognitivos }\end{array}$ & $\begin{array}{l}\text { Competencia } \\
\text { relacionada con } \\
\text { el ítem }\end{array}$ \\
\hline $\begin{array}{l}\text { Estructura de los } \\
\text { textos literarios: } \\
\text { - Inicio } \\
\text { - Desarrollo } \\
\text { (nudo y clímax) } \\
\text { - Final }\end{array}$ & $\begin{array}{l}\text { Uso de plataforma } \\
\text { virtual y sus } \\
\text { herramientas: } \\
\text { calendario, foros, } \\
\text { chats, correo } \\
\text { electrónico, } \\
\text { autoevaluaciones, } \\
\text { acceso a } \\
\text { materiales. } \\
\text { Programas para } \\
\text { elaboración de } \\
\text { historietas. }\end{array}$ & $\begin{array}{l}\text { Aprendizaje por } \\
\text { proyecto: } \\
\text { presentación de } \\
\text { una historieta. } \\
\text { Aprendizaje } \\
\text { colaborativo. }\end{array}$ & $\begin{array}{l}\text { Conocimiento y } \\
\text { comprensión de } \\
\text { la estructura a } \\
\text { través de la } \\
\text { lectura de textos. } \\
\text { Análisis de } \\
\text { textos. } \\
\text { Crea una } \\
\text { historieta con la } \\
\text { estructura } \\
\text { literaria. }\end{array}$ & $\begin{array}{l}\text { A través de la } \\
\text { historieta pudiste } \\
\text { identificar la } \\
\text { estructura del } \\
\text { texto narrativo. }\end{array}$ \\
\hline $\begin{array}{l}\text { Formas de } \\
\text { expresión: } \\
\text { - Diálogo } \\
\text { - Monólogo } \\
\text { - Exposición } \\
\text { - Narración }\end{array}$ & $\begin{array}{l}\text { Uso de plataforma } \\
\text { virtual y sus } \\
\text { herramientas. }\end{array}$ & $\begin{array}{l}\text { Aprendizaje por } \\
\text { proyecto: } \\
\text { presentación de } \\
\text { una historieta } \\
\text { agregando otros } \\
\text { elementos. } \\
\text { Aprendizaje } \\
\text { colaborativo. }\end{array}$ & $\begin{array}{l}\text { Evalúa la } \\
\text { historieta } \\
\text { elaborada. } \\
\text { Integra formas de } \\
\text { expresión dentro } \\
\text { de las viñetas. }\end{array}$ & $\begin{array}{lr}\text { A través del } \\
\text { empleo de la } \\
\text { historieta } & \text { se } \\
\text { identificaron las } \\
\text { características } & \text { de } \\
\text { las formas } & \text { de } \\
\text { expresión. } & \\
\text { En la historieta se } \\
\text { emplearon las } \\
\text { formas } \\
\text { expresión. }\end{array}$ \\
\hline $\begin{array}{l}\text { Figuras literarias: } \\
\text { - Comparación } \\
\text { - Metáfora } \\
\text { - Epíteto } \\
\text { - Paradoja } \\
\text { - Hipérbole }\end{array}$ & $\begin{array}{l}\text { Uso de plataforma } \\
\text { virtual y sus } \\
\text { herramientas: } \\
\text { calendario, foros, } \\
\text { chats, correo } \\
\text { electrónico, } \\
\text { autoevaluaciones, } \\
\text { acceso a } \\
\text { materiales y } \\
\text { presentaciones. } \\
\text { Videos, audios } \\
\text { (canciones)yQuizi } \\
\text { zz }\end{array}$ & $\begin{array}{l}\text { Analogías, } \\
\text { adivinanzas. }\end{array}$ & $\begin{array}{l}\text { Selección de } \\
\text { audios, } \\
\text { canciones. } \\
\text { Analiza la letra y } \\
\text { elige figuras } \\
\text { literarias. } \\
\text { Evalúa la } \\
\text { estructura de } \\
\text { acuerdo con la } \\
\text { forma (prosa, } \\
\text { verso, estrofa) } \\
\text { Crea una } \\
\text { composición. }\end{array}$ & $\begin{array}{l}\text { Los recursos } \\
\text { digitales } \\
\text { empleados te } \\
\text { permitieron la } \\
\text { identificación de } \\
\text { las figuras } \\
\text { literarias. } \\
\text { Los recursos } \\
\text { digitales } \\
\text { empleados te } \\
\text { permitieron la } \\
\text { clasificación de } \\
\text { las figuras } \\
\text { literarias. }\end{array}$ \\
\hline
\end{tabular}




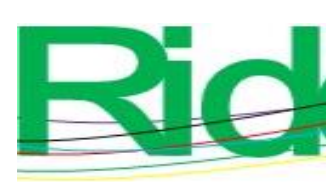

Revista Iberoamericana para la Investigación y el Desarrollo Educativo

ISSN 2007 - 7467

\begin{tabular}{|c|c|c|c|c|}
\hline $\begin{array}{l}\text { Niveles de } \\
\text { análisis literario: } \\
\text { - Informativo } \\
\text { - Ideológico } \\
\text { - Estilístico }\end{array}$ & $\begin{array}{l}\text { Uso de plataforma } \\
\text { virtual y sus } \\
\text { herramientas: } \\
\text { calendario, foros, } \\
\text { chats, correo } \\
\text { electrónico, } \\
\text { autoevaluaciones, } \\
\text { acceso a } \\
\text { materiales: } \\
\text { presentaciones, } \\
\text { cortometrajes y } \\
\text { textos breves: } \\
\text { narrativos, } \\
\text { dramáticos y } \\
\text { líricos. }\end{array}$ & $\begin{array}{l}\text { Impulso de } \\
\text { actividades de } \\
\text { investigación } \\
\text { documental. } \\
\text { Aprendizaje por } \\
\text { proyectos: } \\
\text { - Infografía }\end{array}$ & $\begin{array}{l}\text { Análisis del } \\
\text { material por } \\
\text { niveles. } \\
\text { Emite juicio } \\
\text { valorativo sobre } \\
\text { la obra y su } \\
\text { contexto. } \\
\text { Crea una } \\
\text { infografía del } \\
\text { análisis literario. }\end{array}$ & $\begin{array}{l}\text { A través de los } \\
\text { recursos } \\
\text { empleados se } \\
\text { realizó el análisis } \\
\text { por niveles de las } \\
\text { obras literarias. } \\
\text { El análisis } \\
\text { literario fue } \\
\text { empleado para la } \\
\text { reflexión de la } \\
\text { obra. }\end{array}$ \\
\hline
\end{tabular}

Fuente: Elaboración propia

Así pues, siguiendo lo dispuesto en la tabla 1, en las dos primeras sesiones se les explicó a los participantes en qué consistía la metodología de flipped classroom, haciendo hincapié en la importancia de la revisión del material antes de la sesión presencial disponible en la plataforma denominada Classroom. También se les informó que habría otros recursos de tipo digital, tales como videos, lecturas, blogs, material interactivo de acceso libre en Internet para la realización de ejercicios y evaluaciones, material que, además, de manera inmediata, arroja resultados de la evaluación para retroalimentar, profundizar en los temas y aclarar dudas, se realizó una práctica de exploración del material.

Posteriormente, durante cada una de las sesiones presenciales, los primeros minutos se orientaron hacia el contenido de la plataforma y revisado por los alumnos a través de preguntas detonadoras, ejercicio o esquema para dar paso a las actividades prácticas, que llevaron a la elaboración de breves proyectos en equipo y en el aula (los que están señalados en la columna de la dimensión psicopedagógica). La clase concluyó con la evaluación, sugerencias y comentarios hacia el trabajo realizado.

Es necesario aclarar que las competencias comunicativas están implícitas en el programa de estudios de Expresión Oral y Escrita II. Además, que dentro del mapa curricular del nivel medio son consideradas dentro de las competencias genéricas, aplicables en diversos contextos, relevantes para todas las disciplinas y transferibles, tanto el trabajo en equipo como la expresión y la comunicación en distintos contextos y el empleo de códigos, herramientas y medios o canales apropiados. 


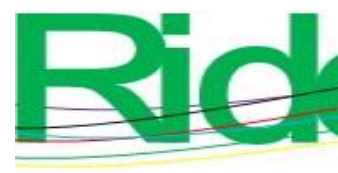

Revista Iberoamericana para la
Investigación y el Desarrollo Educativo
ISSN $2007-7467$

Es en las actividades en el aula en donde se desarrollan las habilidades comunicativas; se orientaron a fomentar el aprender hablando, solucionando retos, discutiendo para la toma de decisiones, de acuerdo con lo que implica la situación comunicativa por resolver, por ejemplo, el diseño de una historieta (involucra la secuencia de la estructura de un texto narrativo a través de dibujos) y las actividades lúdico expresivas asociadas a los juegos, como es el caso de las adivinanzas y el empleo de analogías, con el propósito de fomentar con facilidad el escuchar, la lectura, la expresión oral y escrita, así hacer de las competencias comunicativas un proceso permanente y gradual en su complejidad.

Aunado a todo ello, se realizaron los diferentes tipos de evaluación por el momento, intención y participación de los involucrados: diagnóstica, formativa, sumativa, autoevaluación y coevaluación, empleando técnicas e instrumentos acordes a cada fin.

\section{Instrumento}

El instrumento para recopilar la información fue el cuestionario. Se trató de uno integrado por tres dimensiones: tecnológica, psicopedagógica y de prácticas de uso. Dicho instrumento se formuló mediante una escala de Likert con cinco niveles para los ítems: "Muy frecuente", "Frecuentemente", “Ocasionalmente", "Raramente" y "Nunca". Una vez formulado el cuestionario inicial, se efectuó la validación del contenido a través de la técnica de juicio experto, con el fin de corroborar tanto la congruencia como la redacción de cada ítem y posteriormente llevar a cabo las modificaciones necesarias.

De la misma manera, se indagó la consistencia interna del instrumento a través del coeficiente alfa de Cronbach. Y como resultado se obtuvo un valor de 0.878 , por lo que se interpretó como confiable. Su aplicación fue de forma presencial, explicando el objetivo y relevancia del estudio a los participantes, garantizando la confidencialidad por la información obtenida, la cual, posteriormente, se analizó a través de la estadística descriptiva. 


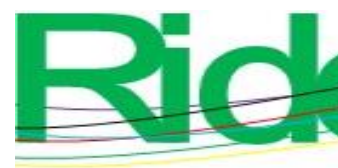

Revista Iberoamericana para la Investigación y el Desarrollo Educativo ISSN 2007 - 7467

\section{Resultados}

Para contar con elementos que dieran certeza del éxito del presente estudio se identificaron los dispositivos electrónicos con que los alumnos disponen. Se encontró que solo $3 \%$ usa la tableta, $6 \%$ utilizan la laptop, $86 \%$ el celular y $92 \%$ la computadora de escritorio. Asimismo, se halló que $28 \%$ no cuenta con un dispositivo inteligente, no dispone de datos o no posee conexión a internet. En estos últimos casos se ofreció apoyo al alumno y se le permitió que entregara las evidencias en el momento y la forma en que pudiera (manuscritas, fotos, correos), solicitándoles la continua y permanente comunicación.

Por otra parte,en cuanto a la dimensión tecnológica (figura 2), la mayoría de los participantes manifestaron que la implementación de la estrategia flipped classroom dio la oportunidad de trabajar con recursos informáticos. Dentro de esta categoría se integraron los mismos elementos considerados en la planeación; aquí se encontró que muy frecuentemente se usó la plataforma virtual y sus herramientas tales como el calendario, foros, chats, correo

electrónico y autoevaluaciones (92\%); programas para elaboración de historietas y presentaciones (87\%); cortometrajes y películas (96\%); canciones (90\%), y la realización de autoevaluaciones (88 \%). Por otra parte, como gamificación se empleó el Quizizz (92 \%) $\mathrm{y}$, finalmente, la lectura de textos $(88 \%)$.

Este hallazgo confirma el resultado obtenido por Schmeisser y Medina (2018) en cuanto a la efectividad de la metodología flipped classroom al usar recursos tecnológicos por parte de los estudiantes. Además, coincide con lo encontrado por Simon et al. (2018) en cuanto a que los materiales didácticos tienen que ser diversos para promover una participación activa en los alumnos y que habría que considerar múltiples formatos y respetar el estilo de aprendizaje de cada uno de los alumnos. 


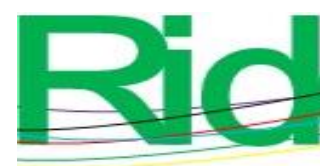

Revista Iberoamericana para la Investigación y el Desarrollo Educativo ISSN $2007-7467$

Figura 2. Dimensión tecnológica

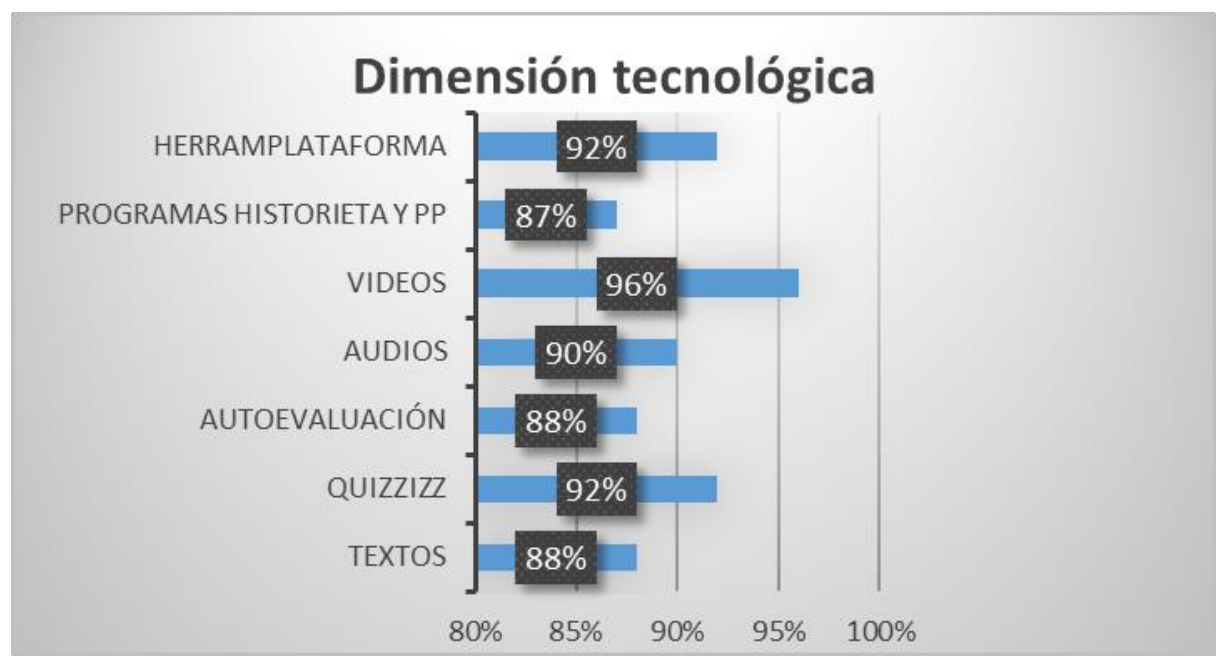

Fuente: Elaboración propia

En lo que respecta a la dimensión psicopedagógica (figura 3), la cual consideró el uso de recursos de diseño instruccional, se integraron metodologías y estrategias activas como aprendizaje por proyectos y colaborativos, realizados por equipos de cuatro o cinco integrantes. Así, las actividades que más frecuentemente se realizaron fueron: elaboración de historieta e infografías (96\%); empleo de analogías (98\%); adivinanzas (100\%); gamificación (92\%), e investigación documental (88 \%).

Estos datos confirman lo hallado por Melo y Sánchez (2017) en lo referente a que el flipped classroom incentiva el trabajo colaborativo, propicia un aprendizaje experiencial, así como la realización de actividades prácticas y fáciles de relacionar con los contenidos a desarrollar en la asignatura. 


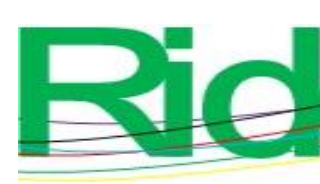
Revista Iberoamericana para la
Investigación y el Desarrollo Educativo
ISSN $2007-7467$

Figura 3. Dimensión psicopedagógica

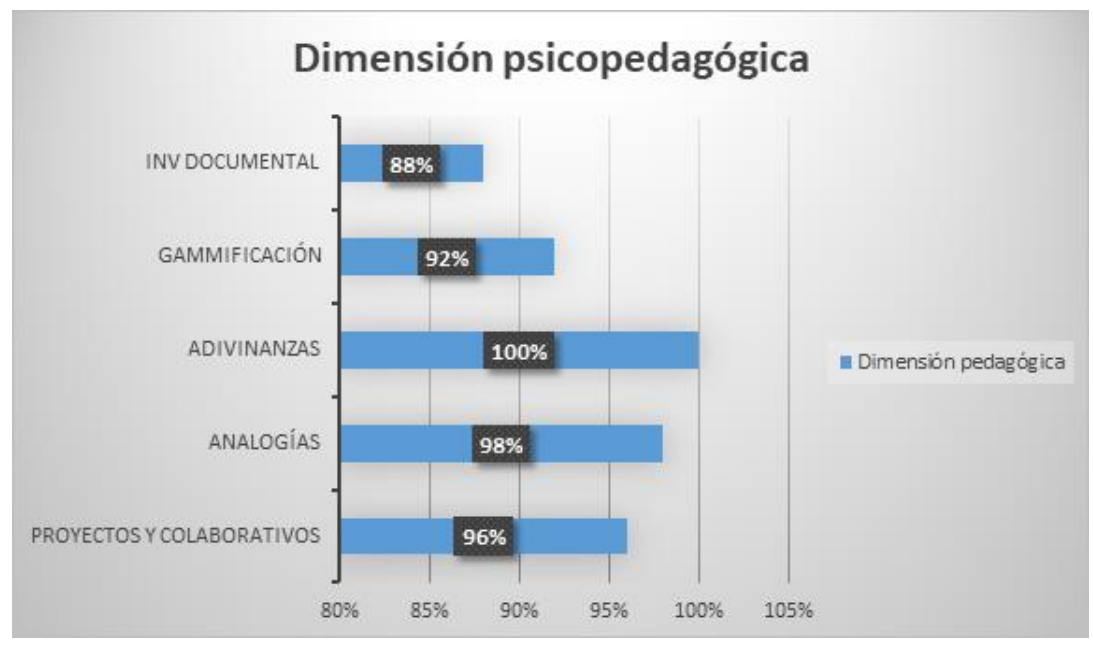

Fuente: Elaboración propia

Por último, la dimensión relacionada con las prácticas de uso, cuya característica principal se relaciona con la unidad I, por ejemplo, los contenidos temáticos empleados en las estrategias centradas en los estudiantes y los productos considerados como evidencias integradoras de los proyectos colaborativos; los resultados obtenidos (figura 4) en la categoría "Muy frecuente" fueron los siguientes: conocimiento y comprensión de la estructura del texto literario (96\%); creación de una historieta con la estructura literaria (100 \%); evaluación de la estructura de la historieta elaborada (100\%); integración de formas de expresión dentro de las viñetas (96 \%); selección de audios y canciones para analizar las letras y figuras literarias (96\%); elaboración de una composición (90\%); análisis del material (cortometraje, video y textos) por niveles (90\%); emisión de juicios valorativos sobre la obra y su contexto (96 \%), y creación de una infografía del análisis literario (100 \%).

De este modo, los resultados permiten corroborar el alcance de los niveles de conocimiento de orden superior como son aplicación, análisis, síntesis y evaluación , así como el logro la competencia particular y los resultados esperados de aprendizaje. Este hallazgo ratifica lo encontrado por García y Cremades (2019) en lo que respecta a que el flipped classroom promueve el proceso de aprendizaje en sus diversos niveles establecidos: recordar, aplicar, analizar y evaluar para alcanzar el de crear. 


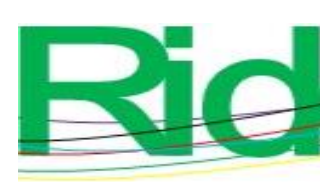

Revista Iberoamericana para la Investigación y el Desarrollo Educativo ISSN $2007-7467$

Figura 4. Prácticas de uso

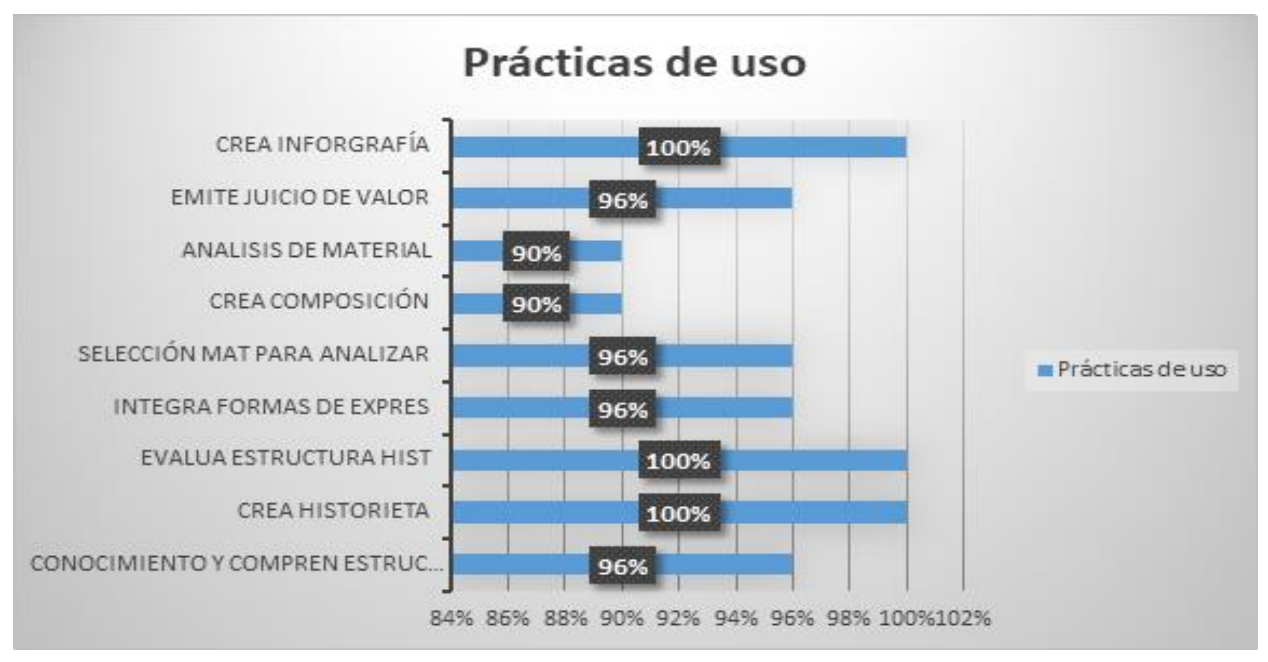

Fuente: Elaboración propia

A manera de cierre de este apartado, se confirma que la implementación del flipped classroom concretó el empleo y desarrollo de la dimensión tecnológica por medio del empleo de las herramientas tecnológicas para la consulta y elaboración de historietas, videos, audios, autoevaluaciones, quizzes y textos. Es clara la tendencia por parte del alumnado de aceptar las actividades realizadas en el aula señaladas en la dimensión psicopedagógica, sobre todo el empleo de adivinanzas ,analogías y proyectos colaborativos.

Referente a la dimensión de práctica, los mayores porcentajes tienden al agrado o aceptación por crear una historieta y evaluarla, así como de crear infografías. De las tres dimensiones, esta es la que tiene el mayor porcentaje de aceptación, la que permite la práctica de la expresión, el análisis, la síntesis y la creatividad.

\section{Discusión}

Los resultados de esta investigación confirman la relevancia de incorporar en el nivel medio superior metodologías activas como el flipped classroom que permitan que el alumno participe de forma protagónica en la construcción de las competencias en un entorno de trabajo colaborativo, tal y como lo mencionan Álvarez et al. (2010).

De la misma forma, es importante la integración y uso continuo de las TIC en los procesos de aprendizaje del estudiante para el logro de los aprendizajes esperados de cada nivel educativo. Además, el flipped classroom permite al alumno la capacidad de identificar, analizar, discernir y seleccionar información para la realización de cualquier actividad formativa (Álvarez y Boillos, 2015). 


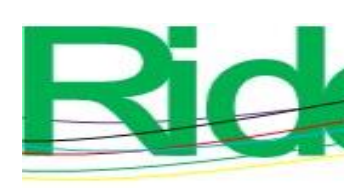

Revista Iberoamericana para la Investigación y el Desarrollo Educativo ISSN $2007-7467$

- $\quad$ Finalmente, es de vital importancia verificar que los alumnos cuenten con los equipos, dispositivos y conectividad, que tengan acceso a los materiales y plataformas en casa o en otros espacios, de lo contrario será una estrategia selectiva y no una inclusiva.

\section{Conclusiones}

El aula invertida oflipped classroom es una estrategia didáctica que plantea, como su nombre lo dice, invertir o cambiar las tareas o trabajos que se realizan de manera tradicional: lo realizado en casa hacerlo en clase y viceversa: poner en práctica en el aula y en compañía de un profesor como mediador los conocimientos adquiridos en casa, donde los recursos tecnológicos pueden ser de gran ayuda.

La implementación de la estrategia flipped classroom requiere de la creación y búsqueda de materiales para trabajar en espacios fuera del aula. Para ello es necesario que el docente dedique tiempo para el diseño e identificación de recursos didácticos digitales que sean susceptibles de ser empleados en la unidad de aprendizaje y así obtener el máximo beneficio de ellos como medios de apoyo en el desarrollo de las competencias. Además, es relevante que el profesorado domine las estrategias o metodologías activas para poderlas implementar en el aula, así motivar y fomentar un aprendizaje significativo que incluya procesos de orden superior o complejo como el pensamiento crítico, la creatividad y el trabajo colaborativo. Por tanto, implica que el trabajo docente vaya mucho más allá de una clase magistral, la cual se suele orientar solamente a las habilidades receptivas de los niveles cognitivos bajos, tales como recordar, repetir o memorizar.

Definitivamente, el empleo de la tecnología y la estrategia flipped classroom promueve los niveles de aprendizaje complejos: motiva el desarrollo de habilidades del pensamiento crítico desde el momento en que los alumnos reflexionan, analizan, toman decisiones, se comunican de manera eficaz (a través de diversos canales y códigos) y emplean la creatividad para el diseño y elaboración de evidencias parciales y productos finales.

Por consiguiente, aquí las competencias comunicativas desarrolladas a través del empleo de la flipped classroom fueron la expresión escrita, oral, la lectura y la escucha, las cuales se formaron por el intercambio de ideas expresadas como puntos de vista individuales o de equipo a través de exposiciones, diálogo, discusión, exploración o investigación y argumentación con el uso de diversos canales y códigos de comunicación . 


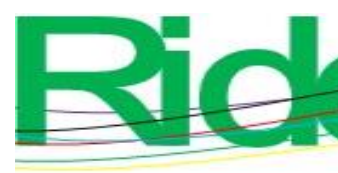

Revista Iberoamericana para la Investigación y el Desarrollo Educativo ISSN 2007 - 7467

Así, la estrategia flipped classroom permitió desarrollar algunas de las habilidades necesarias para que el alumno sea capaz de hacerle frente a situaciones del mundo real: el uso de la comunicación, la creatividad y el trabajo colaborativo.

\section{Futuras líneas de investigación}

Considerando los resultados del estudio, es necesario continuar la investigación del conocimiento y práctica de los docentes sobre el uso de las metodologías activas, ya que no es suficiente la revisión de los materiales proporcionados a los alumnos previo a las sesiones. En este sentido, es relevante analizar el tipo de actividades y estrategias implementadas durante las sesiones para que los alumnos alcancen niveles complejos de conocimiento. Por tanto, se sugiere utilizar como estudio futuro el enfoque cualitativo, a fin de obtener e interpretar los testimonios de los estudiantes con respecto a su experiencia en las metodologías activas.

Asimismo, se recomienda llevar a cabo otras investigaciones acerca de la implementación del flipped classroom en el nivel medio superior en otras unidades de aprendizaje. Del mismo modo, ampliar el horizonte del nivel educativo en el IPN y considerar al ámbito superior, licenciatura y posgrado.

Otro elemento importante para investigar es el alcance de los recursos tecnológicos disponibles por parte de los alumnos, ya que se presupone que en general disponen de los recursos mínimos, como son el acceso y conectividad a un dispositivo tecnológico, cuando en la realidad se han detectado casos desafortunados de estudiantes que no cuentan con ello.

Finalmente, se sugiere realizar estudios sobre los logros alcanzados por esta metodología activa a fin de cuestionarse si es el flipped classroom o el tipo de abordaje práctico en el aula o las estrategias empleadas lo que tiene mayor impacto en los aprendizajes del alumnado. También, se recomienda llevar a cabo investigaciones sobre la valoración del profesorado acerca del empleo de metodologías didácticas basadas en las TIC.

\section{Agradecimientos}

Los autores agradecen al Instituto Politécnico Nacional (IPN) las facilidades otorgadas para la realización de este estudio a partir de la autorización del proyecto de investigación: SIP20200758 titulado "Evaluación del impacto de la formación académica de los posgrados en administración” y del proyecto 20211263 titulado "Aula invertida para el desarrollo de habilidades blandas en la unidad de aprendizaje de expresión Oral y Escrita I" 


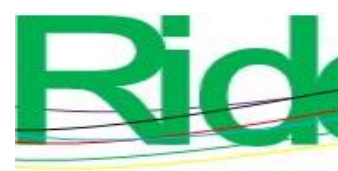
Revista Iberoamericana para la
Investigación y el Desarrollo Educativo
ISSN $2007-7467$

\section{Referencias}

Álvarez, M. y Boillos, M. (2015). La competencia comunicación escrita. En Villardón, L. (coord. $\left.{ }^{\mathrm{a}}\right)$, Competencias genéricas en educación superior. Metodologías específicas para su desarrollo (pp. 55-78). España: Narcea Universitaria.

Álvarez, V. Herrejón, V., Morelos, M. y Rubio, M. (2010). Trabajo por proyectos: aprendizaje con sentido. Revista Iberoamericana de Educación, 52(5), 1-13.

Bondad, B. A., Rice, R. E. and Pearce, K. E. (2012). Influences on TV Viewing and Online User-Shared Video Use: Demographics, Generations, Contextual Age, Media Use, Motivations, and Audience Activity. Journal of Broadcasting \& Electronic Media, 56(4), 471-493.

Calvo, A. (6 de febrero de 2017). Metodologías activas y flipped classroom. Recuperado de https://www.theflippedclassroom.es/metodologias-activas/.

Constitución Política de los Estados Unidos Mexicanos. (2020). Diario Oficial de la Federación. Recuperado

de http://www.diputados.gob.mx/LeyesBiblio/pdf/1_280521.pdf.

Del Moral, C. (2012). Conocimiento didáctico general para el diseño y desarrollo de experiencias de aprendizaje significativas en la formación del profesorado. Profesorado. Revista de Currículum y Formación de Profesorado, 16(2), 421-452. Recuperado de https://recyt.fecyt.es/index.php/profesorado/article/view/43811.

Domínguez, F. y Palomares, A. (2020). El aula invertida como metodología activa para fomentar la centralidad en el estudiante como protagonista de su aprendizaje. Contextos Educativos. Revista de Educación, (26), 261-275. Recuperado de https://doi.org/10.18172/con.4727.

García, D. y Cremades, R. (2019). "Flipped classroom" en educación superior. Un estudio a través de relatos de alumnos. Revista Mexicana de Investigación Educativa, 24(80), 101-123.

García, M. y Quijada, V. (2015). El aula invertida y otras estrategias con uso de TIC. Experiencia de aprendizaje con docente. Ponencia presentada en el Simposio Virtual Internacional Somece 2015. Ciudad de México, del 11 al 13 de noviembre de 2015. Recuperado de http://somece2015.unam.mx/MEMORIA/57.pdf. 


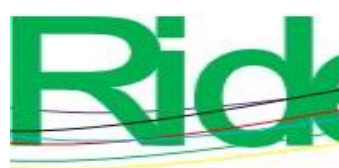

Revista Iberoamericana para la
Investigación y el Desarrollo Educativo
ISSN $2007-7467$

Gilboy, M. B., Heinerichs, S. and Pazzaglia, G. (2015). Enhancing Student Engagement Using the Flipped Classroom. Journal of Nutrition Education and Behavior, 47(1), 109-114. Retrieved from http://dx.doi.org/10.1016/j.jneb.2014.08.008.

Hernández, R. y Mendoza, C. (2018). Metodología de la investigación. Las rutas cuantitativa, cualitativa y mixta. México: McGraw-Hill.

Hinojo, F., Mingorance, A., Trujillo, J., Aznar, I. and Cáceres, M (2018). Incidence of the Flipped Classroom in the Physical Education Student's Academic Performance in University Contexts. Sustainability, 10(5), 1-13. Retrieved from https://doi.org/10.3390/su10051334.

Huang, J., Chen, R. and Wang, X. (2012). Factors influencing intention to forward short internet videos. Social Behavior and Personality, 40(1), 5-14.

Instituto Politécnico Nacional [IPN]. (2008). Programas de estudios de la Unidad de Aprendizaje: Expresión Oral y Escrita II. México: Instituto Politécnico Nacional.

Konopka, C., Adaime, M. and Mosele, P. (2015). Active Teaching and Learning Methodologies: Some Considerations. Creative Education, 6(14), 1536-1545.

López, M., León, M. y Pérez, P. (2018). El enfoque por competencias en el contexto universitario español. La visión del profesorado. Revista de Investigación Educativa, 36(2), 529-545. Recuperado de http://dx.doi.org/10.6018/rie.36.2.314351.

Martínez, G., Nolla, N., Vidal, M. y de la Torre, L. M. (2016). Los entornos personales de aprendizaje en los procesos de formación formales e informales. Educación Médica Superior, 30(3), 599-608. Recuperado de http://scielo.sld.cu/scielo.php?script=sci_arttext\&pid=S086421412016000300013\&l $\mathrm{ng}=\mathrm{es} \& \operatorname{tlng}=\mathrm{es}$.

Martínez, W., Esquivel, I. y Martínez, J. (2014). Aula invertida o modelo invertido de aprendizaje: origen, sustento e implicaciones. En Esquivel, I. (coord.), Los modelos tecno-educativos, revolucionando el aprendizaje del siglo XXI (pp. 143-160). México: DSAE-Universidad Veracruzana

Melo, L. y Sánchez, R. (2017). Análisis de las percepciones de los alumnos sobre la metodología flipped classroom para la enseñanza de técnicas avanzadas en laboratorios de análisis de residuos de medicamentos veterinarios y contaminantes. Educación Química, 28(1), 30-37. Recuperado de http://dx.doi.org/10.1016/j.eq.2016.09.010. 


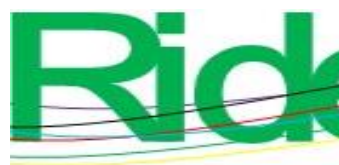

Revista Iberoamericana para la
Investigación y el Desarrollo Educativo
ISSN $2007-7467$

Mendoza, J. (2019). Hoy tenemos el poder de la transformación del proceso educativo: Ana Lidia Franzoni Velázquez. Docencia Politécnica, 1(1), 10-12.

Namakforoosh, J. M. (2011). Metodología de la investigación. México: Limusa.

Navaridas, F. y Jiménez, M. (2016). Concepciones de los estudiantes sobre la eficacia de los ambientes de aprendizaje universitarios. Revista de Investigación Educativa, 34(2), 503-519. Recuperado de http://dx.doi.org/10.6018/rie.34.2.239481.

Núñez, K. y González, J. (2019). Perfil de egreso doctoral: una propuesta desde el análisis documental y las expectativas de los doctorandos. IE Revista de Investigación Educativa de la Rediech, 10(18), 161-175. Recuperado de http://dx.doi.org/10.33010/ie_rie_rediech.v10i18.604.

Organización de las Naciones Unidas para la Educación, la Ciencia y la Cultura [Unesco]. (2016). Declaración de Incheon y Marco de Acción para la realización del Objetivo de Desarrollo Sostenible 4.4 Recuperado de https://unesdoc.unesco.org/ark:/48223/pf0000245656_spa.

Pienta, N. (2016). A "Flipped Classroom" Reality Check. Journal of Chemical Education, 93(1), 1-2. Retrieved from https://pubs.acs.org/doi/10.1021/acs.jchemed.5b00996.

Presidencia de la República. (30 de septiembre de 2019). Ley General de Educación. Diario Oficial de la Federación. Recuperado de https://www.sep.gob.mx/work/models/sep1/Resource/558c2c24-0b12-4676-ad908ab78086b184/ley_general_educacion.pdf.

Rangel,M y Quijada, V (2015). El aula invertida y otras estrategiascon uso de las TIC. Experiencia de aprendizaje con docentes. XXX Simposium SOMEC Recuperado de http://somece2015.unam.mx/MEMORIA/57.pdf

Santos, J., Simões, A. and Vieira, M. (2019). Innovative pedagogical practices in higher education: An integrative literature review. Nurse Education Today, 72, 12-17.

Schmeisser, C. M. y Medina, J. A. (2018). Estudio comparativo entre metodología de aula invertida y metodología tradicional en clases de español, inglés y matemáticas. $M L S$ Educational Research, 2(2), 159-176.

Silva, J. y Maturana, D. (2017). Una propuesta de modelo para introducir metodologías activas en educación superior. Innovación Educativa, 17(73), 117-131. Recuperado de http://www.scielo.org.mx/scielo.php?script=sci_arttext\&pid=S166526732017000100117\&lng=es\&tlng=es. 

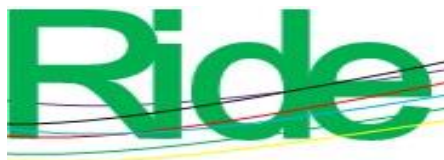

Revista Iberoamericana para la Investigación y el Desarrollo Educativo ISSN $2007-7467$

Simon, J., Ojando, E., Avila, X., Miralpeix, A., Lopez, P. y Prats, M. (2018). Reformulación de los roles del docente y del discente en la educación. El caso práctico del modelo de la Flipped Classroom en la universidad. Revista de Estudios y Experiencias en Educación, (número especial 2), 53-73.

Subsecretaría de Educación Media Superior. (2019). La nueva escuela mexicana: principios y orientaciones pedagógicas. Ciudad de México, México: Secretaría de Educación Pública. http://dfa.edomex.gob.mx/sites/dfa.edomex.gob.mx/files/files/NEM\%20principios\% 20y\%20orientacio\%C3\%ADn\%20pedago\%C3\%ADgica.pdf.

Verleur, R., Heuvelman, A. and Verhagen, P. (2011). Trigger videos on the Web: Impact of audiovisual design. British Journal of Educational Technology, 42(4), 573-582. Retrieved from https://doi.org/10.1111/j.1467-8535.2010.01065.x.

Vidal, M., Rivera, N., Nolla, N., Morales, I. y Vialart, M. N. (2016). Aula invertida, nueva estrategia didáctica. Educación Médica Superior, 30(3), 678-688. Recuperado de http://scielo.sld.cu/scielo.php?script=sci_arttext\&pid=S0864-

21412016000300020\&lng=es\&tlng=es. 


\begin{tabular}{|l|l|}
\hline Rol de Contribución & Autor (es) \\
\hline Conceptualización & $\begin{array}{l}\text { María Elena Zepeda Hurtado(igual), Edgar Oliver Cardoso } \\
\text { Espinosa (igual) y Jésica Alhelí Cortes Ruíz (igual) }\end{array}$ \\
\hline Metodología & $\begin{array}{l}\text { Edgar Oliver Cardoso Espinosa (igual) y Jésica Alhelí Cortes } \\
\text { Ruíz (igual) }\end{array}$ \\
\hline Software & Edgar Oliver Cardoso Espinosa \\
\hline Validación & Jésica Alhelí Cortes Ruíz \\
\hline Análisis Formal & $\begin{array}{l}\text { María Elena Zepeda Hurtado (igual) y Jésica Alhelí Cortes } \\
\text { Ruíz (igual) }\end{array}$ \\
\hline Investigación & $\begin{array}{l}\text { María Elena Zepeda Hurtado (igual), Edgar Oliver Cardoso } \\
\text { Espinosa (igual) y Jésica Alhelí Cortes Ruíz (igual) }\end{array}$ \\
\hline Recursos & $\begin{array}{l}\text { María Elena Zepeda Hurtado (igual), Edgar Oliver Cardoso } \\
\text { Espinosa (igual) y Jésica Alhelí (igual) }\end{array}$ \\
\hline Curación de datos & Edgar Oliver Cardoso Espinosa \\
\hline $\begin{array}{l}\text { Escritura - Preparación del } \\
\text { borrador original }\end{array}$ & $\begin{array}{l}\text { María Elena Zepeda Hurtado (igual), Edgar Oliver Cardoso } \\
\text { Espinosa (igual) y Jésica Alhelí Cortes Ruíz (igual) }\end{array}$ \\
\hline $\begin{array}{l}\text { Escritura - Revisión y } \\
\text { edición }\end{array}$ & $\begin{array}{l}\text { María Elena Zepeda Hurtado (igual), Edgar Oliver Cardoso } \\
\text { Espinosa (igual) y Jésica Alhelí Cortes Ruíz (igual) }\end{array}$ \\
\hline Visualización & María Elena Zepeda Hurtado \\
\hline Supervisión & Edgar Oliver Cardoso Espinosa \\
\hline Administración de Proyectos & $\begin{array}{l}\text { María Elena Zepeda Hurtado (igual), Edgar Oliver Cardoso } \\
\text { Espinosa (igual) y Jésica Alhelí Cortes Ruíz (igual) }\end{array}$ \\
\hline
\end{tabular}

